\title{
NOTA GOTICA
}

Consideriamo questi casi: ${ }^{1}$

jah saei sandida mik atta, sah weitwodeip bi mik

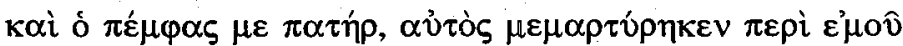

(Giovanni, 5, 37 = Skeireins 5, 17-8);

appan hwa taujis pu taikne?

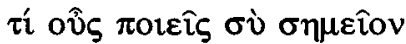

(Giovanni, 6, 30);

sai, jau ainshun pize reike galaubidedi imma aippau Fareisaie?

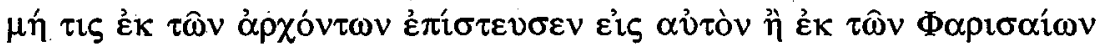
(Giovanni, 7, 48 = Skeireins, 8, 15);

piudinassaus is ni wairpip andeis

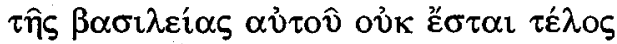

(Luca, 1, 33);

ip Maria alla gafastaida bo waurda

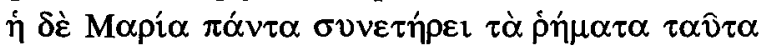

(Luca, 2, 19);

dauhtar ainoho was imma swe wintriwe twalibe

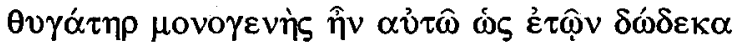

(Luca, 8, 42);

juka auhsne usbauhta fimf

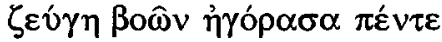

(Luca, 14, 19)

hwan managans habaip hlaibans?

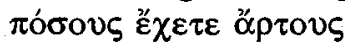

(Marco, 8, 5);

widuwo gawaljaidau ni mins saihs tigum jere

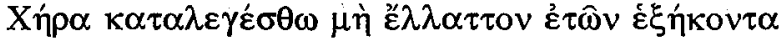

(Lettera a Timoteo I, 5, 9).

1 Tutte le citazioni sono da STREITBERG. 
Dalla documentazione presentata, ancorché in modo non completo, si può constatare che ogni qual volta il testo greco presenta il verbo nella posizione 'in incastro', anche in quello gotico troviamo la stessa situazione. E benché crediamo a una sostanziale dipendenza dal greco dalla prassi sintattica gotica, ${ }^{3}$ tuttavia riteniamo che non si possa del tutto escludere di trovarsi in presenza di uno stesso tratto sintattico, cioè di uno stesso modo di distribuire l'informazione, secondo un preciso ordine dei costituenti di frase, di origine indoeuropea. La "spezzatura di ciò che secondo il nostro sentimento linguistico è unito [...] è comune sia tra i Greci che tra i Latini e gli Indiani ${ }^{4}$ e si trova in tracce ben riconoscibili anche nell'epica germanica". 5 Purtroppo, come già G. Bonfante, anche noi pur "scandagliando l'epopea germanica [non abbiamo] trovato nulla in questo senso". ${ }^{6} \mathrm{La}$ presenza sicura di questo tratto nel gotico però e, per chi come noi gli dà valore, $l^{\prime}$ uso della tmesi ${ }^{7}$ in testi epici germanici ${ }^{8}$ sono lí ad avvalorare l'ipotesi che la posizione 'in incastro' del verbo rappresenta 'il modo più antico, facilmente $c$ omprensibile $\mathrm{d}$ al $\mathrm{p}$ unto di $\mathrm{v}$ ista psicologico, di ordinare le parole nella frase idg". 9 Ė ben vero che il passo del Vangelo di Luca $(2,25)$ " $\pi v \varepsilon \hat{v} \mu \alpha \hat{\hat{\eta}} \nu$ ơ $\gamma$ rov é $\pi^{\prime}$ $\alpha$ $\tau o ́ v "$, reso in gotico "ahma weihs was ana imma", sembrerebbe opporsi a quanto è stato appena affermato. Ma cosí non è. Infatti, "il ne faut pas perdre de vue que Wulfila a suivi un manuscrit grec du type de ${ }^{*} \mathrm{~K}$ ou $* \mathrm{~K}^{\mathrm{L}}$, mais que la version gotique présente des leçons propre à $\mathrm{I}^{\alpha \delta 5 "} .{ }^{10} \mathrm{E}$ molti manoscritti appartenenti al 'Tipo I' ${ }^{11}$ riportano la lezione " $\pi v \varepsilon \hat{v} \mu \alpha$ ơ $\gamma$ เov $\hat{\eta} v " .12$ Pertanto, l'unica conclusione che da questo esempio si deve trarre è che il grande Vescovo dei Goti abbia avuto sotto gli occhi un testo di questa ultima famiglia e, come è nel suo stile, ne sia stato condizionato.

2 Intendiamo quella collocazione particolare che viene ad assumere una parola quando, interponendosi tra altre due strettamente connesse, le separa e, almeno apparentemente, ne spezza l'unità. L'espressione 'in incastro' è stata utilizzata da $\mathrm{G}$. Bonfante, per evitare il termine 'iperbato' che, secondo lui e con qualche ragione "si presta ad equivoci"' (BONFANTE, p. 14).

3 Come ritiene la stragrande maggioranza degli studiosi. Una opinione diversa espressa in KLEIN.

4 Per una trattazione del problema con ampia esemplificazione da queste (e altre) lingue, cfr. BonfaNTE.

5 SCHULZE, col. 1472 .

6 BONFANTE, p. 14

7 Si parla di tmesi quando "due morfemi o sintagmi che l'uso grammaticale unisce strettamente si trovano separati da elementi intercalati" (GRUPPO $\mu$, p. 124). Non c' è dubbio, però, che tale figura retorica diventi così, né più né meno, una forma generale dell'iperbato". (Mortara Garavelli, p. 300), anzi, G. R. Cardona (CARDONA, s.u.) al lemma tmesi, rinvia, senz'altra spiegazione, a quello di iperbato. A sua volta, l'iperbato, almeno come fatto sintattico, "non è sempre chiaramente distinto nelle esemplificazioni classiche" (MORTARA Garavelli, p. 230) dall'anastrofe. Come si vede, dunque, la tmesi e l'iperbato sopra tutto si collocano entrambi tra "quelle modificazioni nell'ordine dei costituenti di frase che sono dovute a variazioni nel 'distribuire' l'informazione" (Ibidem). Forse è per questo che G. Bonfante (cfr. n. 2) ha preferito il sintagma 'posizione «in incastro»' a quello di 'iperbato'.

8 Per esempio, cfr. "[...] gurtun sih iro suert ana" (Hildebrandslied, $\left.5^{b}\right)$; “[...] da stuont ouch Hagene b b" (Niebelungenlied, 1665, $\left.2^{\mathrm{b}}\right) ;$ " $[\ldots]$ do blihte si in an" (Niebelungenlied, 1665, $3^{\mathrm{b}}$ ).

9 SCHULZE, col. 1472.

10 Cuendet, p. 2.

11 Cfr. StReitberg, p. xxxix.

12 Cuendet, p. 29. 


\section{Bibliografia}

BONFANTE 1929: G. Bonfante, Contributi glottologici. II. La posizione «in incastro» del verbo finito idg., Roma, Scuola di filologia classica dell'Università di Roma, s. I, fasc. iv, Roma, 1929, pp. 14-36.

CARDONA 1988: G. R. Cardona, Dizionario di linguistica, Roma, Armando, 1988.

CUENDET 1929: G. Cuendet, L'ordre des mots dans le texte grec et dans les versions gotique, arménienne et vieux slave des Évangiles. I. Les groups nominaux, Paris, Edouard Champion, 1929.

Garavelli Mortara 1988: B. Garavelli Mortara, Manuale di retorica, Milano, Edizioni CDE, 1988.

GRUPPO $\mu$ 1976: Gruppo $\mu$, Retorica generale. Le figure della comunicazione, Milano, Bompiani, 1976.

KLEIN 1992: J. S. Klein, On the Independence of Gothic Syntax. I: Interrogativity, Complex Sentence Types, Tense, Mood, and Diathesis, "Journal of Indo-European Studies" 20 (1992), pp. 339-379.

SchUlze 1890: A. Schulze, rec. a R. Meister, Die griechischen Dialekte, auf Grundlage von Ahrens Werk 'De graecae linguae dialectis', 2. Band, Göttingen, 1890, "Berliner philologische Wochenschrift", 10 (1890 [ma 1891]), coll. 1469-1475.

StreitBerg 2000: W. Streitberg [mit einem Nachtrag von P. Scardigli], Die gotische Bibel. I, Der gotische Text und seine griechische Vorlage, Heidelberg, Winter, $2000^{7}$.

\section{Povzetek}

\section{OPOMBA K ZGRADBI SINTAGME V BIBLIJSKI GOTŠČINI}

Primerjava zgradbe stavkov $v$ evangelijih Nove zaveze med grškim izvirnikom in Wulfilovim prevodom $v$ gotščino kaže, da se gotski prevod sklada $z$ izvirnikom $v$ za skladnjo nenavadnem, presenetljivem, stilističnem urejanju sintagme, na splošno imenovanem hiperbaton. 